\title{
Metabolic Vipaka
}

\author{
Dayakshi D. K. Abeyaratne ${ }^{1}$ Gagan Priya ${ }^{2} \quad$ Sanjay Kalra ${ }^{3} \quad$ Than Than Aye ${ }^{4}$ Moe Wint Aung \\ Manilka Sumanatilleke ${ }^{1}$
}

${ }^{1}$ Diabetes and Endocrinology Unit, National Hospital of Sri Lanka, Sri Lanka

2Department of Endocrinology, Fortis Hospital, Mohali, India

${ }^{3}$ Department of Endocrinology, Bharti Hospital, Karnal, India

${ }^{4}$ Department of Endocrinology, University of Medicine 2,

Yangon, Myanmar

${ }^{5}$ Department of Endocrinology, University of Medicine 1,

Yangon, Myanmar

Address for correspondence Sanjay Kalra, MD, DM, Department of Endocrinology, Bharti Hospital, Karnal 132001, India

(e-mail: bridekn!@gmail.com).

J Soc Health Diab 2019;7:28-30

\author{
Abstract \\ Keywords \\ - biopsychosocial \\ diabetes management \\ - metabolic karma \\ - metabolic vipaka \\ - mindfulness \\ meditation
}

Diabetes management should focus on biopsychosocial aspects and comprehensive care. The philosophical tenets of Buddhism can be a source of inspiration for both the individual with diabetes and health care provider. Buddhism preaches the immutable relationship between action and outcomes. This has a corollary in diabetes care; optimized metabolic control in diabetes is associated with improved short- and long-term outcomes and a legacy effect. Buddhism emphasizes on maintaining a "middle path." When applied to diabetes care, this would mean avoiding both extremes of hyperglycemia and hypoglycemia and optimization of metabolic health while minimizing treatment-emergent adverse events. Mindfulness meditation-based stress reduction strategies may further improve not only psychological health but also biophysical outcomes in diabetes and merit further research.
Buddhism is a way of life, which has provided guidance and wisdom to millions of people across the world over centuries. The philosophical tenets of Buddhism are a source of inspiration and motivation for people from all walks of life. Detailed discussion on the relevance of this timeless religion to persons living with diabetes and their health care providers has been explored in earlier publications. ${ }^{1,2}$

In recent years, diabetes care has evolved into an evidence-based, patient-centered, outcome-oriented, multifaceted discipline. The objectives of diabetes care include not only amelioration of symptoms and biochemical abnormalities but also psychosocial health and improved quality of life. More importantly, diabetes care focuses on prevention of long-term complications and attainment of optimal long-term outcomes. This is ensured by using interventions and strategies that are proven to offer cardiovascular and microvascular or renal safety and benefit. This proof is available from cardiovascular outcome trials $\mathrm{s}^{3,4}$ and follow-up of long-term trials that demonstrate the existence of a metabolic memory or glycemic legacy. ${ }^{5-7}$

Hyperglycemic symptoms improve with only modest improvement in glycemic control, and most individuals with diabetes remain asymptomatic. In addition, the results of long-term metabolic control are not immediately gratifying and difficult to comprehend. This often leads to suboptimal adherence to treatment and inadequate follow-up. Therefore, while significant strides are being made in the pharmacologic approaches in diabetes management, improving long-term patient adherence poses a formidable challenge. Cultural and religious beliefs are inherent to most societies and bear a huge influence on many communities. Culture- and religion-specific communication and motivation may help address the gaps in patient-provider communication..$^{1,2,8-11}$

Buddhism preaches the immutable relationship between action (karma, Sinhala and Sanskrit; kamma, Pali; kan, Myanma) and outcomes (vipaka, Sinhala, Sanskrit, and Pali; aakyoe, Myanma). Good deeds or actions lead to good results or outcomes. This is especially true if these deeds are performed with a good or selfless intention (kusal karma, Sinhala; su-karma, Sanskrit; Kuthol kan, Myanma). ${ }^{12,13}$

This concept, which is a central pillar of Buddhism, influences all aspects of day-to-day life. This belief, or value system, can be extrapolated to diabetes care as well. Good metabolic karma/kamma/kan refers to multifactorial diabetes received

December 6, 2018 accepted after revision

December 11, 2018
DOI https://doi.org/

10.1055/s-0039-1683468 ISSN 2321-0656.
(C)2019 Novo Nordisk Education Foundation
License terms

(요 (1) $\odot \circledast$ 
management, using effective, safe, and well-tolerated drugs to improve outcomes. ${ }^{12,13}$

It is further explained in Buddhist teachings that the karmic seeds (beeja, Sinhala; bija, Sanskrit and Pali; ah saet in Myanma) that are sown today may sprout either immediately or may bear results after a lag period. While Buddhism, like most other religions of the East such as Hinduism, Jainism, and Sikhism, proposes the philosophy of transgenerational karma, the effects of karma/kamma/ kan may be cultivated in the present lifetime itself and this has been referred to as dittha dhamma vedaniya karma (Sinhala, Pali, Myanma).

This has a corollary in diabetes care, because the accumulation of good metabolic karma/kamma leads to good metabolic vipaka/aakyoe, in the form of both short- and long-term health. This metabolic memory or legacy has been demonstrated in long-term outcome trials in both types 1 and 2 diabetes with relation to the effects of improved glycemic control and multifactorial risk factor management. ${ }^{14,15}$ In addition, there may be an early or delayed appearance of cardiovascular benefit with some interventions in diabetes.

Such an explanation of the importance of timely and appropriate diabetes care is rational and respectful of the culture of our community. It facilitates an understanding of why a particular therapeutic approach has been suggested by the diabetes care provider and why expected results may not always be obvious in the short term. It also emphasizes the need for appropriate action by the person living with diabetes, such as self-care, lifestyle modification including proper diet, proper exercise, and healthy habits, as well as stress management. Adherence to therapy and active participation in the management of diabetes are also covered in the ambit of metabolic karma/kamma/kan.

Buddhism plays a key emphasis on the five precepts (pan sil, Sinhala; pancasila, Pali and Sanskrit; ngar par thi la, Myanma). The fifth precept focuses on avoidance of alcohol or other intoxicating substances including tobacco. This bears importance in diabetes management as substance abuse in diabetes may contribute to increased risk for complications and suboptimal control.

In his very first discourse at Sarnath, Gautama Buddha described the "middle path" (madhum piliwetha, Sinhala; madhyam pratipad, Pali; majjhima patipada, Sanskrit). This remains a core concept in modern-day Buddhist philosophy. When applied to diabetes care, this would mean that we must adopt a middle path, avoiding both extremes of hyperand hypoglycemia. Pharmacologic treatment should aim at optimization of metabolic health, but simultaneously steering away from treatment-emergent adverse events. In addition, stress has a strong bidirectional relationship with diabetes. Although diabetes itself can lead to significant distress in persons with diabetes and their families, stress further contributes to suboptimal self-care and metabolic control. The teachings of Buddhism inspire persons with diabetes to avoid either extreme of self-deprivation or overindulgence, while living a healthy balanced and fulfilling life.

Acute anxiety and chronic stress are known to cause a surge in counterregulatory hormones and affect diabetes control. Stress reduction and mental relaxation have been recognized as a means of improving targets and self-care behavior. Meditation has been considered an important tenet in the Buddhist practice. The aim is to develop mindfulness, concentration, and insight. The role of meditation in stress management and diabetes care has been explored in several studies ${ }^{16}$ Although initial smaller studies have been encouraging, more research is required in this field.

Diabetes is a disorder that affects not only the individual but the family and the community as well. Therefore, the management of diabetes requires active participation of all stakeholders. The concept of "for the benefit of the other" (an ayage yahapatha wenuwen, Sinhala) reminds us of our shared responsibilities in tackling this modern-day epidemic.

If the diabetes care provider practices good metabolic karma/kamma, in a timely manner, his or her patients will be able to attain and maintain good metabolic vipaka/aakyoe. This, in Buddhist theory, allows him or her to earn merit (pin, Sinhala; punna, Pali; punya, Sanskrit) or the collection of spiritual currency that results in having a good life. By adhering to a healthy lifestyle and diabetes therapy, the person living with diabetes also earns merit. Thus, a win-win situation is created, which allows accumulation (and potential transfer) of merit.

Through this letter, we have shared the concept of metabolic karma/kamma and metabolic vipaka/aakyoe in diabetes care. We hope that this knowledge, shared across the globe, will help in accumulation of both physical health and spiritual pin/punna/punya.

\section{Conflict of Interest}

None declared.

\section{References}

1 Kalra S, Priya G, Grewal E, et al. Lessons for the health care practitioner from Buddhism. Indian J Endocrinol Metab 2018;22(6):812-817

2 Kalra S, Priya G, Grewal E, et al. Diabetes management and the Buddhist philosophy: toward holistic care. Indian J Endocrinol Metab 2018;22(6):806-811

3 Schnell O, Rydén L, Standl E, Ceriello A; D\&CVD EASD Study Group. Updates on cardiovascular outcome trials in diabetes. Cardiovasc Diabetol 2017;16(1):128

4 John M, Gopalakrishnan Unnikrishnan A, Kalra S, Nair T. Cardiovascular outcome trials for anti-diabetes medication: a holy grail of drug development? Indian Heart J 2016;68(4):564-571

5 Ihnat MA, Thorpe JE, Ceriello A. Hypothesis: the 'metabolic memory', the new challenge of diabetes. Diabet Med 2007;24(6):582-586

6 Chalmers J, Cooper ME. UKPDS and the legacy effect. N Engl J Med 2008;359(15):1618-1620

7 Thomas MC. Glycemic exposure, glycemic control, and metabolic karma in diabetic complications. Adv Chronic Kidney Dis 2014;21(3):311-317

8 Kalra B, Joshi A, Kalra S, et al. Coping with Illness: insight from the Bhagavad Gita. Indian J Endocrinol Metab 2018;22(4):560-564

9 Kalra S, Joshi A, Kalra B, et al. Bhagavad Gita for the physician. Indian J Endocrinol Metab 2017;21(6):893-897

10 Priya G, Kalra S, Dardi IK, et al. Diabetes care: inspiration from Sikhism. Indian J Endocrinol Metab 2017;21(3):453-459

11 Niazi AK, Kalra S. Patient centered care in Islam: distinguishing between religious and sociocultural factors. J Diabetes Metab Disord 2013;12(1):30 
12 Kalra S, Ved J, Baruah MP. Diabetes destiny in our hands: Achieving metabolic karma. Indian J Endocrinol Metab 2017;21(3):482-483

13 Kalra S, Kawatra P. Metabolic karma in diabetes care: medico-philosophical reflections. Indian J Endocrinol Metab 2017;21(4):643-644

14 Cooper ME, El-Osta A, Allen TJ, Watson AMD, Thomas MC, Jandeleit-Dahm KAM. Metabolic Karma-the atherogenic legacy of diabetes: The 2017 Edwin Bierman Award Lecture. Diabetes 2018;67(5):785-790
15 Gæde P, Oellgaard J, Carstensen B, et al. Years of life gained by multifactorial intervention in patients with type 2 diabetes mellitus and microalbuminuria: 21 years follow-up on the Steno-2 randomised trial. Diabetologia 2016;59(11):2298-2307

16 Priya G, Kalra S. Mind-body interactions and mindfulness meditation in diabetes. Eur Endocrinol 2018;14(1):35-41 